\title{
Inhaled corticosteroids and survival in chronic obstructive pulmonary disease: does the dose matter?
}

\author{
D.D. $\operatorname{Sin} *$, , S.F.P. Man*
}

\begin{abstract}
Inhaled corticosteroids and survival in chronic obstructive pulmonary disease: does the dose matter? D.D. Sin, S.F.P. Man. C) ERS Journals Ltd 2003.

ABSTRACT: Recent data suggest that inhaled corticosteroids reduce the number of clinical exacerbations in chronic obstructive pulmonary disease (COPD). It remains unknown whether a dose/response relationship exists. The present study was conducted to evaluate the long-term impact of varying doses of inhaled corticosteroids on COPD mortality.

Hospital discharge data were used to identify all patients aged $\geqslant 65$ yrs recently hospitalised due to COPD in Alberta, Canada $(n=6,740)$. The relative risk $(R R)$ for allcause mortality was compared across different dose categories of inhaled corticosteroids (none and low, medium and high doses) following hospital discharge.

Inhaled corticosteroid therapy after discharge was associated with a $25 \%$ relative reduction in risk for all-cause mortality (RR $0.75,95 \%$ confidence interval (CI) 0.68-0.82). Patients on medium- or high-dose therapy showed lower risks for mortality than those on low doses (RR 0.77, 95\% CI 0.69-0.86 for low dose; RR $0.48,95 \%$ CI 0.37-0.63 for medium dose; and RR 0.55, 95\% CI 0.44-0.69 for high dose).

Use of inhaled corticosteroids following hospital discharge for chronic obstructive pulmonary disease was associated with a significant reduction in the overall mortality rate. Low- was inferior to medium- or high-dose therapy in protecting against mortality in chronic obstructive pulmonary disease.
\end{abstract}

Eur Respir J 2003; 21: 260-266.

Chronic obstructive pulmonary disease (COPD) is one of the leading causes of morbidity and mortality in the world and the only major cause of mortality whose incidence is rising [1]. Best estimates suggest that, by the year 2020, COPD will be the fifth leading cause of disability (currently twelfth) [2] and the third leading cause of mortality (currently fourth) if current trends continue [3]. Ironically, COPD is the most underfunded disease in relation to its burden of illness on society [4]

Although some progress has been made in the diagnosis and treatment of COPD during the 1990s, there is a scarcity of therapies that can effectively ameliorate the inevitable decline in lung function or health status of patients suffering from this disorder [5]. Since chronic airway inflammation is a prominent feature of COPD [6], some have suggested that inhaled corticosteroids may improve the health outcomes of COPD patients [7]. Although data to date have been mixed, the totality of evidence suggests that they reduce the number of clinical exacerbations [8] and retard the decline in health status of COPD patients [9] without significantly modifying the rate of decline in forced expiratory volume in one second (FEV1) [9-13].

$\overline{\text { For editorial comment see page } 202 .}$
*Dept of Medicine, Pulmonary Division, University of Alberta, and ${ }^{\#}$ Institute of Health Economics, Edmonton, Canada.

Correspondence: D.D. Sin, 2E4.29 Walter C. Mackenzie Centre, University of Alberta, Edmonton AB T6G 2B7, Canada.

Fax: 17804076384

E-mail: don.sin@ualberta.ca

Keywords: Chronic obstructive pulmonary disease, doses, inhaled corticosteroids, mortality

\section{Received: May 162002}

Accepted after revision: September 9 2002

D.D. Sin is supported by a New Investigator Award from the Canadian Institutes of Health Research, Ottawa, Canada, and a Population Health Investigator Award from the Alberta Heritage Foundation for Medical Research, Edmonton, Canada.
These promising results are counterbalanced by recent data linking inhaled corticosteroids to a variety of complications including oral thrush [8], dysphonia [8], bone demineralization [11, 14], cataract formation $[15,16]$ and glaucoma [17], which generally occur in a dose-dependent fashion. Thus, from a safety perspective, low-dose therapy would be desirable. It remains unclear, however, whether low-dose therapy ( $<500 \mu \mathrm{g} \cdot \mathrm{day}^{-1}$ beclomethasone equivalent) offers similar clinical advantages to medium- or high-dose therapy, as there is a scarcity of published data comparing the effects of different dosing levels of these medications on important clinical end-points such as mortality.

Using a large population cohort of patients with moderate-to-severe COPD (previously hospitalised patients) in the province of Alberta, Canada, the dose/ response relationship of inhaled corticosteroids and mortality rates in COPD was investigated.

\section{Methods}

\section{Study population}

Hospital discharge abstracts were obtained from the Canadian Institute for Health Information (CIHI), 
Ottawa, Canada, through Alberta Health and Wellness, Edmonton, Canada. Information from this database included all separations (discharges, hospital admissions, transfers and deaths) for all people residing in the province of Alberta. For the present study, all residents of Alberta, Canada, aged $\geqslant 65 \mathrm{yrs}$, who had experienced at least one hospitalisation for COPD as the most responsible diagnosis between April 1, 1994 and March 31, 1998, were included. International Classification of Diseases ninth revision (ICD-9) codes 490.x, 491.x, 492.x and 496.x were used to identify these patients [18]. All patients with an admitting diagnosis of asthma (ICD-9 code 493.x) were excluded. Patients who died during the index hospitalisation were also excluded. All hospital visits occurring after the first hospital visit were censored for each study patient in order to avoid double counting.

\section{Drug data}

The CIHI database was merged with the Alberta Blue Cross (Edmonton, Canada) database in order to obtain information on medications dispensed for the study cohort. This was possible because all Alberta residents aged $\geqslant 65$ yrs receive government subsidies for prescription medications. All claims in this database have a unique identification number for the medication, as well as the quantity and date dispensed. For each study patient, use of all pulmonary medications (short-acting $\beta_{2}$-agonists, ipratropium bromide, inhaled and oral corticosteroids and oral theophyllines), including those prescribed at the time of discharge, was determined for the study period. Data concerning medications received before the patient's index hospital visit were censored.

\section{Study design}

The study patients were followed for 3 yrs following discharge from the initial hospitalisation for COPD or until the date of their death, if earlier. Mortality data were obtained from Alberta Vital Statistics (Edmonton, Canada), a government agency that keeps an updated electronic file of all deceased persons in Alberta and includes dates of death. In order to determine the "primary" causes of deaths during the follow-up period for the cohort, discharge abstracts of hospitalisations leading to death were obtained from the CIHI database. All deceased persons with ICD-9 codes 460. $x-519 . x$ in the primary diagnostic field were considered to have died from a pulmonary-specific cause of mortality.

Since Alberta Blue Cross provides data on the quantity of medications dispensed rather than the daily dose, the mean daily dose of inhaled corticosteroids was imputed by determining the total dose of medications dispensed for each patient for the first two prescriptions and dividing the total dose of the first dispensation by follow-up time (in days) between these two doses. The calculated mean dose was rounded to a clinically plausible dose [19]. If, for example, a patient was prescribed beclomethasone at a dose of 200 puffs $\left(250 \mu \mathrm{g} \cdot\right.$ puff $\left.^{-1}\right)$ and the elapsed time between the first and second prescription was 100 days, the calculated mean daily dose would be $500 \mu \mathrm{g}$.

To allow cross-comparisons between different inhaled corticosteroid preparations, all formulations were converted into beclomethasone dipropionate equivalents based on equivalency calculations suggested by the Canadian Asthma Consensus Report [20]. The daily dose was then divided into five mutually exclusive categories: not dispensed and low ( $\leqslant 500 \mu \mathrm{g} \cdot \mathrm{day}^{-1}$ beclomethasone or equivalent), medium $\left(501-1,000 \mu \mathrm{g} \cdot \mathrm{day}^{-1}\right)$, high $\left(>1,000 \mu \mathrm{g} \cdot \mathrm{day}^{-1}\right)$ and indeterminate dose. The last category contained patients who received only one dispensation of inhaled corticosteroids during the follow-up period. Although the mean daily dose could not be calculated for these individuals, they most probably received a mean daily dose lower than that received in the low-dose category.

\section{Other factors}

The effect of comorbid conditions was determined using the CIHI database. A modified Charlson comorbidity score was calculated for each individual patient using the ICD-9 codes in the 15 secondary diagnosis fields. In order to derive this score, the following comorbid conditions were taken into account: congestive heart failure (ICD-9 code 428.x), ischaemic heart disease (ICD-9 codes 410.x and 412.x), peripheral vascular disease (ICD-9 codes 443.9, 441.x, 785.4 and V43.4), cerebrovascular accidents (ICD-9 codes 430.x-438.x), dementia (ICD-9 code 290.x), rheumatological diseases (ICD-9 codes 710.0, 710.1, 710.4, 714.0-714.2, 714.81 and 725.x), peptic ulcer disease (ICD-9 codes 531.x-534.9), diabetes (ICD-9 codes 250.0-250.7), malignancy (ICD-9 codes 140.x-172.9, 174.x-195.8, 196.x-199.1 and 200.x-208.9) and renal (ICD-9 codes 582.x, 583.x, 585.x, 586.x and 588.x) and liver diseases (ICD-9 codes 571.x and 572.x) [21]. A Charlson comorbidity score of zero denotes the absence of any comorbid conditions; higher numbers indicate an increasing burden of comorbidity.

\section{Statistical analysis}

The mean and SD of continuous variables were compared using analysis of variance adjusted for multiple comparisons through Tukey's test. Ordinal and binary variables were compared using a Chisquared test (with an appropriate number of degrees of freedom).

The mortality rates of patients who received and did not receive inhaled corticosteroids during the followup period were compared using the Cox proportional hazards model. In this model, age, sex, Charlson comorbidity score, admission to an intensive care unit (ICU) during the initial hospital stay (yes or no variable) and filling in prescriptions for various pulmonary medications, including short-acting $\beta_{2}$-agonists, ipratropium bromide, oral corticosteroids and oral theophyllines, were controlled for. Age was divided into three ranges: $65-74,75-84$ and $\geqslant 85$ yrs. Similarly, 
the Charlson comorbidity score was categorised into three groups: $0,1-2$ and $\geqslant 3$. In order to increase the validity of the model, a group-corrected prognosis method was used for constructing the adjusted survival curves [22]. A similar Cox proportional model was used to compare adjusted survival (using a groupcorrected prognosis method) among those on no and low-, medium-, indeterminate- and high-dose therapy.

A series of secondary analyses were conducted in order to determine the robustness of the findings. It was reasoned that real differences in the effects of different doses of inhaled corticosteroid therapy should be most obvious when compared in a relatively young and healthy subgroup [23]. Therefore, patients aged 65-74 yrs with no documented comorbid conditions were identified and a similar survival analysis was performed in this group of patients using a Cox proportional hazards model. Confounding by age and comorbid conditions should be minimal in this group of patients [23].

\section{Results}

\section{Study population}

Patients aged $\geqslant 65$ yrs who had experienced at least one hospital visit due to their COPD during the study period $(n=6,740)$ were identified. The mean age of the study population was $76.2 \pm 7.2$ yrs. Of these patients: $3,661(54.3 \%)$ were male; $3,744(55.6 \%)$ had no comorbid conditions; $2,736(40.6 \%)$ had a Charlson comorbidity score of 1 or $2 ; 260(3.9 \%)$ had a Charlson comorbidity score of $\geqslant 3$; and $356(5.3 \%)$ were admitted to an ICU during the initial hospitalisation. The mean duration of follow-up was $31.7 \pm 19.3$ months. Of the patients: $4,725(70.1 \%)$ received at least one dispensation of a short-acting $\beta_{2}$-adrenergic medication; 3,751 (55.6\%) received ipratropium bromide; $3,144(46.6 \%)$ received oral corticosteroids; and 1,950 (28.9\%) received oral theophyllines (table 1).

\section{Inhaled corticosteroid use}

Overall, 3,343 (49.6\%) patients received an inhaled corticosteroid during the study period. Of these patients, $2,011(61.2 \%)$ used low-, $318(9.7 \%)$ medium- and 413 $(12.6 \%)$ high-dose therapy and $601(18.0 \%)$ received an indeterminate dose. There were no significant differences in age, sex and Charlson comorbidity score between those who received and did not receive inhaled corticosteroids. Indeed, there were no significant differences in these parameters across different dose categories. However, patients on high-dose inhaled corticosteroid therapy were more likely to have received other pulmonary medications, including ipratropium bromide, oral corticosteroids and oral theophyllines.

\section{Survival}

During the follow-up period, there were 2,404 $(35.7 \%)$ deaths in the cohort. On crude analysis,

Table 1. - Characteristics of elderly chronic obstructive pulmonary disease patients who received and did not receive inhaled corticosteroids

\begin{tabular}{|c|c|c|c|c|c|c|}
\hline & \multicolumn{5}{|c|}{ Dose } & \multirow[t]{2}{*}{$\mathrm{p}$-value } \\
\hline & None & Low & Medium & High & Indeterminate & \\
\hline Subjects n & 3397 & 2011 & 318 & 413 & 601 & \\
\hline \multicolumn{7}{|l|}{ Age yrs } \\
\hline Mean & $77.4 \pm 7.4$ & $75.2 \pm 6.9$ & $74.3 \pm 6.8$ & $74.5 \pm 6.8$ & $75.7 \pm 6.7$ & 0.001 \\
\hline $65-74$ & 177 (53.6) & $466(55.2)$ & $215(55.4)$ & $216(57.8)$ & $269(44.8)$ & 0.001 \\
\hline $75-84$ & $125(37.9)$ & $313(37.1)$ & $129(33.3)$ & $130(34.8)$ & $243(40.4)$ & 0.002 \\
\hline$>84$ & $28(8.5)$ & $65(7.7)$ & $44(11.3)$ & $28(7.5)$ & $59(9.8)$ & 0.001 \\
\hline \multicolumn{7}{|l|}{ Sex } \\
\hline Male & $1855(54.6)$ & $1112(55.9)$ & $176(55.3)$ & $221(53.5)$ & $297(49.4)$ & 0.139 \\
\hline Female & $1542(45.4)$ & $899(44.7)$ & $142(44.7)$ & $192(46.5)$ & $304(50.6)$ & \\
\hline \multicolumn{7}{|l|}{$\begin{array}{l}\text { ICU stay during } \\
\text { index hospitalisation }\end{array}$} \\
\hline Yes & $163(4.8)$ & $107(5.3)$ & $22(6.9)$ & $27(6.5)$ & $37(6.2)$ & 0.238 \\
\hline No & $3234(95.2)$ & $1904(94.7)$ & $296(93.1)$ & $386(93.5)$ & $564(93.8)$ & \\
\hline \multicolumn{7}{|c|}{ Charlson comorbidity score } \\
\hline Mean & $0.76 \pm 1.14$ & $0.57 \pm 0.91$ & $0.56 \pm 0.85$ & $0.52 \pm 0.86$ & $0.66 \pm 1.14$ & 0.001 \\
\hline 0 & $1731(51.0)$ & $1212(60.3)$ & $188(59.1)$ & $261(63.2)$ & $352(58.6)$ & 0.001 \\
\hline $1-2$ & 1509 (44.4) & 737 (36.7) & $126(39.6)$ & $142(34.4)$ & $222(36.9)$ & 0.001 \\
\hline$\geqslant 3$ & $157(4.6)$ & $62(3.1)$ & $4(1.3)$ & $10(2.4)$ & $27(4.5)$ & 0.002 \\
\hline \multicolumn{7}{|l|}{$\begin{array}{l}\text { Use of other } \\
\text { pulmonary drugs }\end{array}$} \\
\hline$\beta_{2}$-adrenergics & 1554 (45.8) & $1929(95.9)$ & $309(97.2)$ & $401(97.1)$ & $532(88.5)$ & 0.001 \\
\hline Oral steroids & $852(25.1)$ & $1440(71.6)$ & $239(75.2)$ & $300(72.6)$ & $313(52.1)$ & 0.001 \\
\hline Theophyllines & $491(14.5)$ & $975(48.5)$ & $123(38.7)$ & $174(42.1)$ & $187(31.1)$ & 0.001 \\
\hline Ipratropium bromide & $1119(32.9)$ & $1648(82.0)$ & $268(84.3)$ & $340(82.3)$ & $376(62.5)$ & 0.001 \\
\hline
\end{tabular}

Data are presented as $\mathrm{n}(\%)$ or mean \pm SD. The p-values reflect a linear trend across the dose categories (from none to high-dose category). ICU: intensive care unit. 


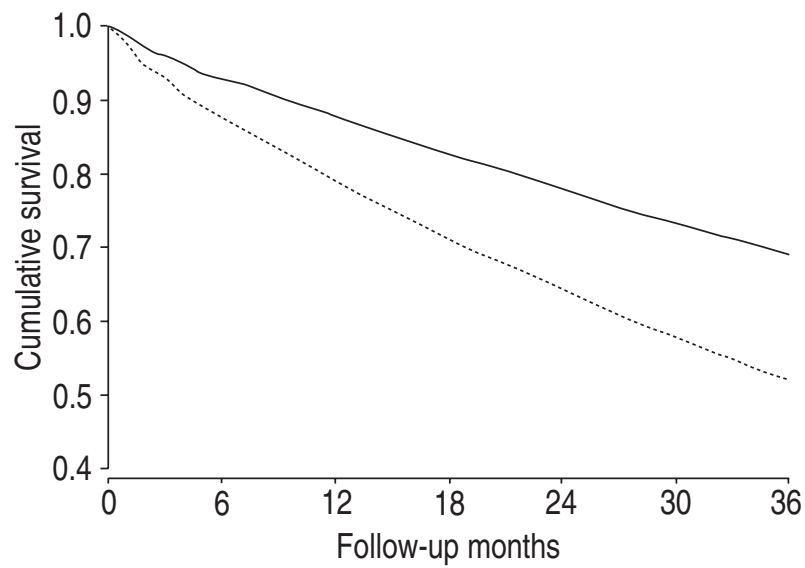

Fig. 1.-Adjusted survival in chronic obstructive pulmonary disease patients who used $(-)$ and did not use $(\cdots \cdots \cdots)$ inhaled corticosteroids following their index hospitalisation. The curves were generated using the group-corrected prognosis method. $\mathrm{p}=0.001$.

therapy with inhaled corticosteroids was associated with $39 \%$ fewer deaths than in those not on these medications (relative risk (RR) $0.61,95 \%$ confidence interval (CI) 0.56-0.66). After adjustments for age, sex, comorbid conditions, ICU stay and use of other pulmonary medications, a $25 \%$ reduction in the overall mortality rate was observed in those who received inhaled corticosteroids compared to those who did not (RR 0.75, 95\% CI 0.68-0.82) (fig. 1).

Patients dispensed low-dose therapy showed a $23 \%$ reduction in mortality rate compared to those who did not receive any inhaled corticosteroids (RR 0.77, 95\% CI $0.69-0.86)$. Those on medium-dose therapy experienced a $52 \%$ reduction (RR $0.48,95 \%$ CI $0.37-0.63$ ) and those on high-dose therapy a $45 \%$ relative reduction (RR $0.55,95 \%$ CI $0.44-0.69$ ) compared to those who did not receive any inhaled corticosteroids (fig. 2). Patients on indeterminate doses did not experience any significant decline in all-cause mortality rate $(\mathrm{R} R$ 0.88, 95\% CI 0.76-1.03; $\mathrm{p}=0.108$ ).

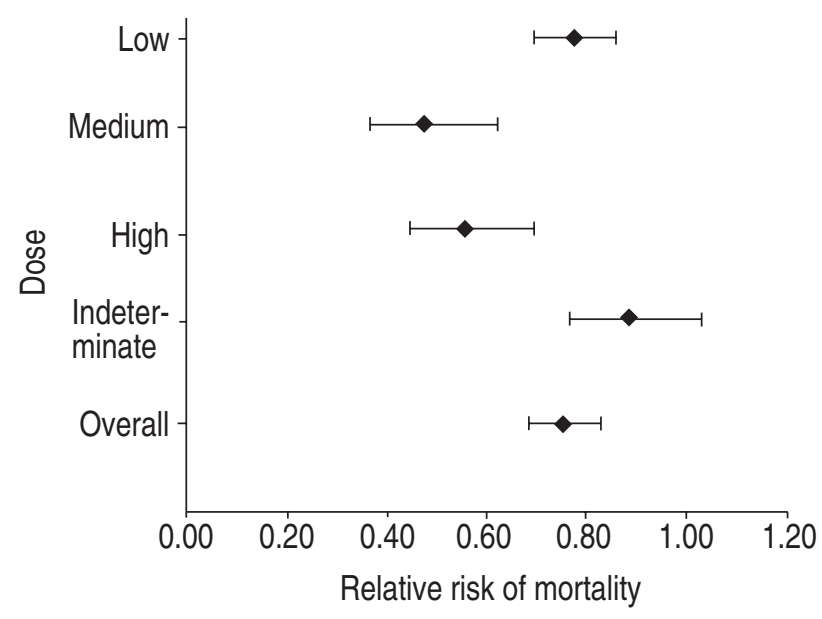

Fig. 2.-Relative risk of all-cause mortality in those in different dose categories who used and did not use inhaled corticosteroids. The reference group did not use any inhaled corticosteroids following hospital discharge. Horizontal bars indicate $95 \%$ confidence interval.
Table 2.-Factors associated with all-cause mortality in chronic obstructive pulmonary disease

\begin{tabular}{|c|c|c|}
\hline Variable & $\mathrm{RR}$ & $95 \%$ CI \\
\hline \multicolumn{3}{|l|}{ Age yrs } \\
\hline $65-74$ & $1.00^{\natural}$ & \\
\hline $75-84$ & 1.35 & $1.23-1.47$ \\
\hline$\geqslant 85$ & 1.84 & $1.64-2.06$ \\
\hline \multicolumn{3}{|c|}{ Charlson comorbidity score } \\
\hline 0 & $1.00^{\circ}$ & \\
\hline $1-2$ & 1.40 & $1.29-1.52$ \\
\hline$\geqslant 3$ & 1.74 & $1.61-1.88$ \\
\hline \multicolumn{3}{|l|}{ ICU stay ${ }^{\#}$} \\
\hline No & $1.00^{\circ}$ & \\
\hline Yes & 1.21 & $1.01-1.44$ \\
\hline \multicolumn{3}{|l|}{ Sex } \\
\hline Female & $1.00^{\circ}$ & \\
\hline Male & 1.37 & $1.25-1.50$ \\
\hline \multicolumn{3}{|c|}{ Inhaled corticosteroids } \\
\hline No & $1.00^{\bullet}$ & \\
\hline Yes & 0.75 & $0.68-0.82$ \\
\hline \multicolumn{3}{|c|}{$\beta_{2}$-adrenergics } \\
\hline No & $1.00^{\top}$ & \\
\hline Yes & 1.23 & $1.09-1.38$ \\
\hline \multicolumn{3}{|c|}{ Oral theophyllines } \\
\hline No & $1.00^{\pi}$ & \\
\hline Yes & 1.07 & $0.97-1.18$ \\
\hline \multicolumn{3}{|c|}{ Ipratropium bromide } \\
\hline No & $1.0^{\bullet}$ & \\
\hline Yes & 1.25 & $1.12-1.39$ \\
\hline \multicolumn{3}{|c|}{ Oral corticosteroids } \\
\hline No & $1.0^{\bullet}$ & \\
\hline Yes & 1.08 & $0.98-1.18$ \\
\hline
\end{tabular}

All analyses have been adjusted for each of the stated variables (see Methods section). RR: relative risk; CI: confidence interval; ICU: intensive care unit. \#: during index hospitalisation; ": reference.

Other risk factors for mortality are shown in table 2, and include advanced age, male sex and a Charlson comorbidity score of $\geqslant 1$. Use of oral theophyllines and oral corticosteroids was not associated with increased mortality. However, there was a slight increase in risk among those who used $\beta_{2}$-adrenergics or ipratropium bromide.

\section{Secondary analyses}

In order to determine the robustness of the inhaled corticosteroid therapy and mortality relationship, a series of subgroup analyses were conducted (table 3 ). Survival benefits of inhaled corticosteroids were observed across different age groups, sexes, and comorbidity and medication statuses.

Even among the healthiest members of the cohort, inhaled corticosteroids were associated with a significant survival advantage. In those aged 65-74 yrs and without any comorbid conditions, it was found that inhaled corticosteroids were associated with a $37 \%$ relative reduction in all-cause mortality rate compared to no therapy (RR $0.63,95 \%$ CI $0.50-0.79$ ). Low dose-therapy was associated with a $37 \%$ reduction (RR 0.63, 95\% CI 0.49-0.82), medium-dose with a $50 \%$ reduction (RR $0.50,95 \%$ CI $0.30-0.83$ ) and 
Table 3.-Relationship between inhaled corticosteroids and all-cause mortality rates in various subgroups of chronic obstructive pulmonary disease patients

\begin{tabular}{|c|c|c|c|c|}
\hline \multirow[t]{2}{*}{ Subgroup } & \multirow[t]{2}{*}{ Overall } & \multicolumn{3}{|c|}{ Corticosteroid dose } \\
\hline & & Low & Medium & High \\
\hline Age $\leqslant 74$ yrs & $0.74(0.63-0.86)$ & $0.74(0.62-0.88)$ & $0.49(0.33-0.71)$ & $0.45(0.31-0.65)$ \\
\hline No ICU stay $\#$ & $0.74(0.67-0.81)$ & $0.76(0.68-0.85)$ & $0.48(0.36-0.63)$ & $0.55(0.43-0.69)$ \\
\hline Short-acting $\beta_{2}$-adrenergic users & $0.63(0.57-0.70)$ & $0.64(0.57-0.72)$ & $0.42(0.32-0.55)$ & $0.46(0.37-0.58)$ \\
\hline Ipratropium bromide users & $0.57(0.51-0.64)$ & $0.59(0.52-0.67)$ & $0.40(0.30-0.53)$ & $0.43(0.33-0.55)$ \\
\hline Oral corticosteroid users & $0.55(0.48-0.63)$ & $0.56(0.48-0.65)$ & $0.36(0.26-0.49)$ & $0.44(0.33-0.57)$ \\
\hline
\end{tabular}

Data are presented as relative risk ( $95 \%$ confidence interval). The reference group did not receive any inhaled corticosteroids during follow-up. All analyses have been adjusted for age, sex, intensive care unit (ICU) stays, Charlson comorbidity score and use of pulmonary medications (i.e. $\beta_{2}$-adrenergics, ipratropium bromide, oral corticosteroids and oral theopylline). "\#. during index hospitalisation.

high-dose with a $57 \%$ reduction $(\mathrm{RR} 0.43,95 \% \mathrm{CI}$ $0.27-0.70)$.

Since survivor treatment selection bias [24] was a potential concern in the analysis, sensitivity analyses were performed including only certain subgroups. For instance, a subgroup analysis was performed excluding all patients who had a follow-up of $\leqslant 90$ days and, therefore, a lower probability of having received inhaled steroids than the rest of the cohort. In this analysis, inhaled corticosteroids were associated with a $43 \%$ lower risk of mortality (RR $0.57,95 \%$ CI $0.51-0.63)$. Using cut-offs of 6,9 and 12 months made little difference to the overall findings (RR 0.57 for 6 months, 0.58 for 9 months and 0.58 for 12 months), suggesting that survivor treatment selection bias was not a significant concern in the analysis.

The impact of inhaled corticosteroids on pulmonary-specific causes of mortality was evaluated. It was found that inhaled corticosteroid therapy was associated with a $30 \%$ risk reduction (RR $0.70,95 \%$ CI $0.53-0.93)$ in pulmonary-specific mortality rate.

\section{Discussion}

By studying a large group of COPD patients recently hospitalised for an acute exacerbation, the present study has produced several interesting and novel findings. First, it has been shown that inhaled corticosteroid therapy was associated with a significant reduction in both all-cause and pulmonaryspecific mortality rates over a 3-yr period. Secondly, medium- and high-dose therapy were associated with greater reductions in mortality rate than low-dose therapy. However, survival benefits were similar between medium- and high-dose therapy.

These findings are supported by the results of a recently published systematic review of all randomised controlled trials, demonstrating the beneficial effects of inhaled corticosteroids on clinical outcomes such as exacerbation rate [8]. Interestingly, these benefits occurred in the absence of any appreciable effect on rate of decline in FEV1 [9-13]. The mechanisms underlying these observations remain largely unknown. Although corticosteroids are less effective at neutralising COPDrelated airway inflammation than that occurring in asthma, several [25-27], but not all [28, 29], studies have demonstrated that therapy with moderate-to-high doses of inhaled corticosteroids for several weeks can significantly attenuate some of the components of airway inflammation in COPD. Inhaled corticosteroids may also attenuate airway hyperresponsiveness [11], a known risk factor for morbidity and mortality in COPD [30].

The present findings are also similar to those of a previous report on patients in Ontario, Canada [18]. In that study, it was demonstrated that inhaled corticosteroids reduced the risk of hospitalisation and allcause mortality over a 12 -month period. However, this study did not evaluate the relationship between mortality and dose of inhaled corticosteroids. The present study extends the earlier findings by demonstrating that the survival benefits of inhaled corticosteroids are maintained for $3 \mathrm{yrs}$. As in the present study, in their meta-analysis, vAN GRUNSVEN et al. [31] showed that high-dose therapy (defined in their study as $\geqslant 1,500 \mu \mathrm{g} \cdot$ day $^{-1}$ beclomethasone or $\geqslant 1,600 \mu \mathrm{g} \cdot$ day $^{-1}$ budesonide) improved flow/volume measurements in lung function tests over time, whereas low doses and placebos did not. In addition, there were no significant differences in the rate of decline in flow/volume measurements between low- and medium-dose therapy. Since important clinical changes can occur in the absence of a discernible effect on flow/volume [9], VAN GRUNSVEN et al. [31] might have missed subtle but important differences in clinical outcome between those on low- and medium-dose therapy. Nevertheless, this systematic review is consistent with the present observation that low-dose therapy is less efficacious than higher doses of inhaled corticosteroids in COPD.

In the present study, a consistent relationship between oral corticosteroid therapy and mortality was not observed. The study of STROM [32] showed that oral corticosteroids were associated with increased risk of hospitalisation in females but not in males. In the study of ScHOLs et al. [33], oral corticosteroid therapy in moderate-to-high doses was associated with increased mortality risk but only in the absence of concomitant inhaled steroid therapy. When combined with inhaled corticosteroids, oral steroid therapy, even at high doses, did not significantly increase the mortality rate [33]. Although it is evident that oral corticosteroids are fraught with many serious adverse effects, they may also produce some clinical benefits 
[34], particularly in the short term. Thus, the benefit/ risk ratio of these medications remains uncertain.

Several limitations of the present study should be addressed. First, no clinical outcomes other than survival were measured. Although mortality is a critically important outcome for assessing the effectiveness of medications, it is by no means the only end-point germane for clinical practice [35]. Health-related quality of life and other morbidity measurements matter to patients and their physicians. The present authors' databases did not contain such information, an important limitation of the present study. Secondly, because baseline lung function data were not available, it was not possible to be certain whether patients receiving low-, medium- or high-dose therapy had similar disease severity. In clinical practice, clinicians generally treat the mildest cases with the lowest doses and reserve high doses for those with either refractory disease or the greatest disease burden. The finding that low-dose therapy is inferior to higher doses of inhaled corticosteroids for reducing mortality risks in COPD is likely to be unchanged by disease severity data. Moreover, a variety of sensitivity analyses were performed and similar results found across all strata, suggesting that the findings are robust and consistent and unlikely to have been confounded by (measured and unmeasured) external factors. Thirdly, if patients who were dispensed inhaled corticosteroids were more likely to have had clinical features of asthma than those who did not receive these medications, the beneficial effect of inhaled steroids would have been overestimated. In order to minimise this bias, all patients who had a primary diagnosis of asthma were excluded from the study. Moreover, the analysis was restricted to the elderly who had required at least one hospitalisation for their disease. Since such patients are very likely to have respiratory failure on the basis of COPD and not asthma, diagnostic bias is unlikely to explain away the present findings [36].

An observational study such as the present one is not intended to replace data from randomised controlled trials, since observational studies are much more susceptible to confounding and bias [37]. Accordingly, the present findings should be interpreted cautiously and conservatively. Nevertheless, these data provide a solid rationale for the systematic evaluation of the effects of inhaled corticosteroids (in varying doses) on hard clinical end-points such as mortality.

In summary, the present study shows a protective benefit of inhaled corticosteroids in mortality that extends to 3 yrs. Moreover, medium- and high-dose therapy are related to improved survival compared to low or indeterminate doses. These data suggest an important role of inhaled corticosteroid therapy in improving clinical outcomes in these high-risk chronic obstructive pulmonary disease patients. They also suggest that, when using inhaled corticosteroids for chronic obstructive pulmonary disease, clinicians should consider using $\geqslant 500 \mu \mathrm{g} \cdot$ day $^{-1}$ beclomethasone (or equivalent) to achieve maximal survival benefits. These results are dissimilar to those in asthma, where low-dose therapy appears to achieve similar clinical outcomes to medium- or high-dose therapy [38]. Clinical trials are needed to confirm these early findings.

\section{References}

1. Hurd S. The impact of COPD on lung health worldwide: epidemiology and incidence. Chest 2000; 117: Suppl. 2, 1S-4S.

2. Michaud CM, Murray CJ, Bloom BR. Burden of disease - implications for future research. JAMA 2001; 285: 535-539.

3. Murray CJ, Lopez AD. Alternative projections of mortality and disability by cause 1990-2020: Global Burden of Disease Study. Lancet 1997; 349: 14981504.

4. Gross CP, Anderson GF, Powe NR. The relation between funding by the National Institutes of Health and the burden of disease. N Engl J Med 1999; 340: 1881-1887.

5. Barnes PJ. Chronic obstructive pulmonary disease. $N$ Engl J Med 2000; 343: 269-280.

6. Jeffery PK. Comparison of the structural and inflammatory features of COPD and asthma. Giles F. Filley Lecture. Chest 2000; 117: Suppl. 1, 251S-260S.

7. Calverley PM. Inhaled corticosteroids are beneficial in chronic obstructive pulmonary disease. Am J Respir Crit Care Med 2000; 161: 341-342.

8. Alsaeedi A, Sin DD, McAlister FA. The effects of inhaled corticosteroids in chronic obstructive pulmonary disease: a systematic review of randomized placebocontrolled trials. Am J Med 2002; 113: 59-65.

9. Burge PS, Calverley PM, Jones PW, Spencer S, Anderson JA, Maslen TK. Randomized double blind, placebo controlled study of fluticasone propionate in patients with moderate to severe chronic obstructive pulmonary disease: the ISOLDE trial. BMJ 2000; 320: 1297-1303.

10. Paggiaro PL, Dahle R, Bakran I, Frith L, Hollingworth $\mathrm{K}$, Efthimiou J. Multicenter randomized placebocontrolled trial of inhaled fluticasone propionate in patients with chronic obstructive pulmonary disease. Lancet 1998; 351: 773-780.

11. The Lung Health Study Research Group. Effects of inhaled triamcinolone on the decline in pulmonary function in chronic obstructive pulmonary disease. $N$ Engl J Med 2000; 340: 1902-1909.

12. Vestbo J, Sorensen T, Lange P, Brix A, Torre P, Viskum K. Long-term effect of inhaled budesonide in mild and moderate chronic obstructive pulmonary disease. Lancet 1999; 353: 1819-1823.

13. Pauwels RA, Lofdahl CG, Laitinen LA, et al. Longterm treatment with inhaled budesonide in persons with mild chronic obstructive pulmonary disease who continue smoking. European Respiratory Society Study on Chronic Obstructive Pulmonary Disease. $N$ Engl J Med 1999; 340: 1948-1953.

14. Wong CA, Walsh LJ, Smith CJ, Wisniewski AF, et al. Inhaled corticosteroid use and bone-mineral density in patients with asthma. Lancet 2000; 355: 1399-1403.

15. Cumming RG, Mitchell P, Leeder SR. Use of inhaled corticosteroids and the risk of cataracts. $N$ Engl J Med 1997; 337: 8-14.

16. Garbe E, Suissa S, LeLorier J. Association of inhaled corticosteroid use with cataract extraction in elderly patients. JAMA 1998; 280: 539-543.

17. Garbe E, LeLorier J, Boivin JF, Suissa S. Inhaled and nasal glucocorticoids and the risks of ocular hypertension or open-angle glaucoma. JAMA 1997; 277: 722-727.

18. Sin DD, Tu JV. Inhaled corticosteroids and the risk of 
mortality and readmission in elderly patients with chronic obstructive pulmonary disease. Am J Respir Crit Care Med 2001; 164: 580-584.

19. Rochon PA, Tu JV, Anderson GM, et al. Rate of heart failure and 1-year survival for older patients receiving low-dose beta-blocker therapy after myocardial infarction. Lancet 2000; 356: 639-644.

20. Boulet LP, Becker A, Berube D, Beveridge R, Ernst P. Canada Asthma Consensus Report, 1999. Canadian Asthma Consensus Group. CMAJ 1999; 161: Suppl. 11, S1-S61.

21. Deyo RA, Cherkin DC, Ciol MA. Adapting a clinical comorbidity index for use with ICD-9-CM administrative databases. J Clin Epidemiol 1992; 45: 613-629.

22. Ghali WA, Quan H, Brant R, et al. Comparison of 2 methods for calculating adjusted survival curves from proportional hazards models. JAMA 2001; 286: 1494-1497.

23. Wen SW, Hernandez R, Naylor CD. Pitfalls in nonrandomized outcomes studies. The case of incidental appendectomy with open cholecystectomy. JAMA 1995; 274: 1687-1691.

24. Glesby MJ, Hoover DR. Survivor treatment selection bias in observational studies: examples from the AIDS literature. Ann Intern Med 1996; 124: 999-1005.

25. Confalonieri M, Mainardi E, Della Porta R, et al. Inhaled corticosteroids reduce neutrophilic bronchial inflammation in patients with chronic obstructive pulmonary disease. Thorax 1998; 53: 583-585.

26. Yildiz F, Kaur AC, Ilgazli A, et al. Inhaled corticosteroids may reduce neutrophilic inflammation in patients with stable chronic obstructive pulmonary disease. Respiration 2000; 67: 71-76.

27. Hattotuwa KL, Gizycki MJ, Ansari TW, Jeffery PK, Barnes NC. The effects of inhaled fluticasone on airway inflammation in chronic obstructive pulmonary disease: a double-blind, placebo-controlled biopsy study. Am J Respir Crit Care Med 2002; 165: 1592-1596.

28. Culpitt SV, Maziak W, Loukidis S, Nightingale JA, Matthews JL, Barnes PJ. Effect of high dose inhaled steroid on cells, cytokines, and proteases in induced sputum in chronic obstructive pulmonary disease. $\mathrm{Am}$ J Respir Crit Care Med 1999; 160: 1635-1639.

29. Cox G, Whitehead L, Dolovich M, Jordana M, Gauldie J, Newhouse MT. A randomized controlled trial on the effect of inhaled corticosteroids on airways inflammation in adult cigarette smokers. Chest 1999; 115: $1271-1277$.

30. Hospers JJ, Postma DS, Rijcken B, Weiss ST, Schouten JP. Histamine airway hyper-responsiveness and mortality from chronic obstructive pulmonary disease: a cohort study. Lancet 2000; 356: 1313-1317.

31. van Grunsven PM, van Schayck CP, Derenne JP, et al. Long term effects of inhaled corticosteroids in chronic obstructive pulmonary disease: a meta-analysis. Thorax 1999; 54: 7-14.

32. Strom K. Oral corticosteroid treatment during longterm oxygen therapy in chronic obstructive pulmonary disease: a risk factor for hospitalization and mortality in women. Respir Med 1998; 92: 50-56.

33. Schols AM, Wesseling G, Kester AD, et al. Dose dependent increased mortality risk in COPD patients treated with oral glucocorticoids. Eur Respir J 2001; 17: 337-342.

34. Bach PB, Brown C, Gelfand SE, McCrory DC. Management of acute exacerbations of chronic obstructive pulmonary disease: a summary and appraisal of published evidence. Ann Intern Med 2001; 134: 600-620.

35. Guyatt GH, Naylor CD, Juniper E, Heyland DK, Jaeschke R, Cook DJ. Users' guides to the medical literature. XII. How to use articles about healthrelated quality of life. Evidence-Based Medicine Working Group. JAMA 1997; 277: 1232-1237.

36. Vestbo J. Another piece of the inhaled corticosteroids in COPD puzzle. Am J Respir Crit Care Med 2001; 164: 514-515.

37. MacMahon S, Collins R. Reliable assessment of the effects of treatment on mortality and major morbidity, II: observational studies. Lancet 2001; 357: 455-462.

38. Adams N, Bestall J, Jones P. Inhaled beclomethasone at different doses for long-term asthma. Cochrane Database Syst Rev 2001; 1: CD002879. 